\title{
Sympathetic Predominance Before Tourniquet Deflation is Associated With a Reduction in Arterial Blood Pressure After Tourniquet Deflation During Total Knee Arthroplasty
}

\author{
Eugene KIM ${ }^{1}$, Myung Rae CHO ${ }^{2}$, Sung Hye BYUN ${ }^{3}$, Jung A LIM $^{4}$, Seungbum CHAE ${ }^{2}$, Won \\ Kee $\mathrm{CHOI}^{2}$, Inyoung $\mathrm{KIM}^{4}$, Jonghae $\mathrm{KIM}^{4}$
}

${ }^{1}$ Department of Anesthesiology and Pain Medicine, Hanyang University Medical Center, College of Medicine, Hanyang University, Seoul, Republic of Korea, ${ }^{2}$ Department of Orthopaedic Surgery, Daegu Catholic University Medical Center, School of Medicine, Daegu Catholic University, Daegu, Republic of Korea, ${ }^{3}$ Department of Anesthesiology and Pain Medicine, Kyungpook National University Chilgok Hospital, School of Medicine, Kyungpook National University, Daegu, Republic of Korea, ${ }^{4}$ Department of Anesthesiology and Pain Medicine, Daegu Catholic University Medical Center, School of Medicine, Daegu Catholic University, Daegu, Republic of Korea

Received January 7, 2021

Accepted March 9, 2021

Epub Ahead of Print May 12, 2021

\section{Summary}

High dependency of arterial blood pressure (ABP) on enhanced sympathetic activity, which maintains vascular tone, leads to hypotension after hemodynamic insults that blunt the sympathetic activity. Therefore, we hypothesized that sympathovagal balance before tourniquet deflation (TD) determines the extent of a reduction in ABP after TD during total knee arthroplasty (TKA). Fifty-four hypertensive female patients undergoing TKA under spinal anesthesia were analyzed. The sympathovagal balance [low-to-high frequency ratio of heart rate variability (LF/HF)] before TD was defined as (LF/HF during 5 min before TD-preanesthetic LF/HF)/preanesthetic LF/HF (\%). An increase in its value represents a shift in sympathovagal balance toward sympathetic predominance. The percent change in the mean ABP (MAP) after TD was defined as (minimum MAP during $10 \mathrm{~min}$ after TD-averaged MAP during $5 \mathrm{~min}$ before TD)/averaged MAP during $5 \mathrm{~min}$ before TD (\%). Simple linear regression was performed to assess the correlation between the sympathovagal balance before TD and change in MAP after TD. The correlation was also assessed by multiple linear regression controlling for age, duration of tourniquet inflation, and spinal anesthesia-induced hypotension. Thirty-two minutes (on average) after tourniquet inflation, the MAP was decreased by 12.1 ( -3.0 to 47.9$) \%$ [mean (range)] upon TD $(P<0.001)$. The sympathovagal balance before TD was negatively proportional to the change in MAP after TD in both simple and multiple linear regression models $\left(R^{2}=0.323\right.$ and $\left.0.340, P<0.001\right)$. A shift in sympathovagal balance toward sympathetic predominance before TD is associated with a decrease in ABP after TD.

\section{Key words}

Arterial pressure - Tourniquets - Total knee arthroplasty • Autonomic nervous system • Hypotension

\section{Corresponding author}

Jonghae Kim, Department of Anesthesiology and Pain Medicine, Daegu Catholic University Medical Center, School of Medicine, Daegu Catholic University, 33, Duryugongwon-ro 17-gil, Nam-gu, Daegu 42472, Republic of Korea. E-mail: usmed12@gmail.com

\section{Introduction}

The sympathetic nervous system regulates arterial blood pressure (ABP) by maintaining vascular tone (Charkoudian and Rabbitts 2009, Joyner et al. 2008, Wallin and Charkoudian 2007). However, interindividual variability (Incognito et al. 2018) exists in the contribution of this system to ABP regulation. For example, the same ABP is achieved in both a person with low sympathetic nervous activity and one with high 
sympathetic nervous activity (Charkoudian et al. 2005). This interindividual variability in sympathetic nervous activity leads to various responses to consistent hemodynamic insults that blunt sympathetic nervous activity (e.g. trimethaphan-induced ganglionic block and spinal anesthesia). The higher the baseline sympathetic nervous activity is, the more $\mathrm{ABP}$ decreases upon hemodynamic insults (Barnes et al. 2014, Furlan et al. 2019, Hanss et al. 2006, Jones et al. 2001), implying that if ABP is highly dependent on tonic sympathetic support, its withdrawal (hemodynamic insults) significantly reduces the $\mathrm{ABP}$. In total knee arthroplasty, $\mathrm{ABP}$ is reduced after tourniquet deflation (TD) due to redistribution of intravascular volume to the ischemic lower limb, hemorrhage, and vasodilatation (Girardis et al. 2000, Gupta et al. 2008). Because the vasodilation is mediated by loss of the sympathetic tone (Sheng and Zhu 2018), we hypothesized that patients with sympathetic predominance prior to TD may have low ABP after TD.

\section{Methods}

\section{Participants}

In this prospective observational study, we enrolled 58 consecutive female patients aged 50-80 years with hypertension (American Society of Anesthesiologists physical status of 2) and a body mass index less than $35 \mathrm{~kg} / \mathrm{m}^{2}$ who were scheduled to undergo unilateral total knee arthroplasty under spinal anesthesia. The present study was approved by the Daegu Catholic University Medical Center Institutional Review Board and conducted in accordance with Good Clinical Practice guidelines and the principles of the Declaration of Helsinki. Before enrollment, the patients provided written informed consent at a preoperative visit. Patients were excluded from the study if they had diabetes mellitus, coronary artery disease, heart failure, cerebrovascular accident, chronic kidney disease, other diseases affecting the autonomic nervous system, coagulation deficiencies, known allergies to local anesthetics, neurological deficits on the operative side, inflammation at the lumbar puncture site, cardiac conduction disorders or arrhythmias, serum electrolyte abnormalities, psychiatric disorders, severe aortic stenosis or mitral stenosis, severe hypovolemia, increased intracranial pressure, or difficulty communicating with medical personnel.

\section{Anesthetic management}

The day before surgery, the patients fasted starting at midnight. Plasmalyte was administered intravenously at a rate of $5 \mathrm{ml} / \mathrm{kg} / \mathrm{hr}$ starting one hour before surgery. No premedication was administered, and antihypertensive drugs were discontinued on the day of surgery (Kaimar et al. 2012). Upon a patient's arrival to the operating room, electrocardiogram (ECG) and pulse oximetry monitoring were implemented. The radial artery of the upper limb ipsilateral to the pulse oximeter probe was catheterized for real-time continuous $\mathrm{ABP}$ monitoring. The pressure transducer was leveled to a position approximately $5 \mathrm{~cm}$ posterior to the sternal border (Courtois et al. 1995). The sensors of a noninvasive cardiac output measurement system (NICOM, Cheetah Medical, Portland, OR, USA) were placed in the bilateral upper and lower regions of the thorax for real-time continuous cardiac index monitoring. The patients remained supine and acclimatized for $10 \mathrm{~min}$ under quiet conditions at ambient temperature to calibrate the NICOM device and subsequently obtain baseline hemodynamic parameters.

After acclimatization, the patients were placed in the lateral decubitus position with the operative side dependent for spinal anesthesia. Following sterilization of the lumbar region with povidone and skin infiltration with $1 \%$ lidocaine $30 \mathrm{mg}$, a 23 -gauge Quincke spinal needle was introduced to the most prominent intervertebral space between L3 and S1. Confirmation of the free flow of cerebrospinal fluid through the needle was followed by the injection of 10-12 mg $0.5 \%$ hyperbaric bupivacaine into the subarachnoid space.

After the induction of spinal anesthesia, the patient was returned to the supine position. Once the sensory block, which was assessed by a pinprick test $20 \mathrm{~min}$ after the injection of bupivacaine, reached the T10 dermatome, surgery was allowed to proceed. Immediately before the beginning of surgery, a sterilized pneumatic tourniquet was applied around the thigh and subsequently inflated to $100 \mathrm{~mm} \mathrm{Hg}$ above the systolic ABP (Odinsson and Finsen 2006) after exsanguination of the leg with an Esmarch bandage. The tourniquet was deflated after soft tissue release following femoral and tibial preparation.

Intraoperative hypotension was defined as a decrease in systolic ABP to below $90 \mathrm{~mm} \mathrm{Hg}$ or a $20 \%$ decrease in the mean ABP (MAP) from the pre-induction baseline. If hypotension developed within $20 \mathrm{~min}$ after the induction of spinal anesthesia, it was defined as hypotension induced by spinal anesthesia. Likewise, hypotension induced by TD was defined as a decrease in 
systolic ABP to below $90 \mathrm{~mm} \mathrm{Hg}$ or a $20 \%$ decrease in the MAP within $10 \mathrm{~min}$ after TD compared with the average value for the $5 \mathrm{~min}$ before TD. Every instance of hypotension was treated with the intravenous administration of $5-10 \mathrm{mg}$ of ephedrine, which was repeated up to three times. All operations and anesthetic management were performed by the same surgeon and anesthesiologist, respectively. All the study cases began and finished between 8:00 am and 12:00 pm.

Measurement of autonomic nervous activity by analysis of heart rate variability

The ECG waveform was continuously recorded at a sampling rate of $1000 \mathrm{~Hz}$ using an MP 100 data acquisition system and AcqKnowledge software (Biopac Systems, Inc., Goleta, CA, USA). Patients were allowed to breathe freely but not to speak or cough during the recording of the ECG waveform. The 5-minute-long waveforms corresponding to each predefined time point were extracted from the ECG waveform using WinDaq Waveform Browser (DATAQ Instruments, Akron, $\mathrm{OH}$, USA). The R peaks of the ECG were detected using Advanced CODAS analysis software (DATAQ Instruments, USA). Erroneously detected peaks and missing or undetected $\mathrm{R}$ peaks were identified by manual inspection and subsequently discarded from and added to the waveform, respectively. The beat-to-beat RR intervals were obtained using the software. The RR intervals of ectopic beats, which were longer or shorter by $20 \%$ than the previous RR interval, were replaced by interpolation from adjacent normal RR intervals. ECG recordings including more than three ectopic beats were excluded from the analysis.

In a tachogram, data were resampled at $4 \mathrm{~Hz}$ from the linearly interpolated values and detrended by performing linear regression. The tachogram was divided into five 100-second-long segments with two adjacent segments overlapping by $50 \%(50 \mathrm{~s})$. Fast Fourier transform was performed in each segment after Hamming windowing (Harris 1978) to obtain the corresponding periodograms, which, in turn, were averaged (Welch 1967). By integrating the area of the averaged periodogram between 0.04 and $0.15 \mathrm{~Hz}$, between 0.15 and $0.4 \mathrm{~Hz}$, and between 0 and $0.4 \mathrm{~Hz}$, the low-frequency power (LF), high-frequency power (HF), and total power of heart rate variability (HRV), respectively, were calculated. The LF represents the combined parasympathetic and sympathetic modulation of the heart rate by baroreflex activity, while the HF represents the parasympathetic modulation of the heart rate in response to spontaneous respiration (Misek et al. 2020). Therefore, the low-to-high frequency ratio $(\mathrm{LF} / \mathrm{HF})$ can be used as an index of the sympathovagal balance (Heart Rate Variability 1996). An increase in its value denotes a shift in the sympathovagal balance toward sympathetic predominance, and vice versa. Using each whole ECG waveform from one patient who developed no hypotension after TD and another who developed hypotension after TD, two HRV spectrograms were made to present the longitudinal change in HRV during the entire surgery. The whole waveform was divided into 100 -second-long segments, which were overlapped by $50 \%$ (50 s). Each segment underwent Fast Fourier transform and the power spectra were presented in decibel $(\mathrm{dB})$ by log-transform of the amplitude obtained by Fast Fourier transform $\left[20 \times \log _{10}(\right.$ amplitude $\left.)\right]$. The spectrogram was plotted with time as abscissa and frequency as ordinate. Power in $\mathrm{dB}$ was displayed as an image with the rainbow colormap. DADiSP software version 6.7 (DSP Development Corp., Newton, MA, USA) with advanced DSP module was used to obtain the power spectral density of HRV and draw the spectrogram.

\section{Outcome measurements}

An independent investigator who was not involved in the anesthetic management collected the study data. The patients' demographic and perioperative data [age, side of surgery, anesthesia data, and duration of tourniquet inflation (TI) and surgery] were collected.

The HRV parameters (LF, HF, LF/HF, and total power) were calculated from the 5-minute-long ECG waveforms obtained during $5 \mathrm{~min}$ before the lateral decubitus position for spinal anesthesia (T1), between 15 and $20 \mathrm{~min}$ after the end of local anesthetic injection into the subarachnoid space (T2), between 10 and $15 \mathrm{~min}$ after TI (T3), during 5 min before TD (T4), and between 5 and $10 \mathrm{~min}$ after TD (T5). The sympathovagal balance before TD was defined as

$$
\frac{L F / H F \text { for } T 4-L F / H F \text { for } T 1}{L F / H F \text { for } T 1}(\%)
$$

Hemodynamic parameters (heart rate, systolic ABP, diastolic ABP, MAP) from the vital sign monitor (CARESCAPE Monitor B850, GE HealthCare, Helsinki, Finland) were recorded at a 10 -second interval using S5 collect software and UPI-PC Serial Cable (GE HealthCare, Finland). At the end of the surgery, the 
hemodynamic data measured by the NICOM throughout surgery, including the cardiac index measured at a 30 -second interval, were retrieved using the NICOM
EMR application version 1.5.5 (Cheetah Medical, USA). The total peripheral resistance index (TPRI) was calculated based on the following formula:

$$
\frac{M A P \times 80}{\text { cardiac index }}\left(\text { dyne } \cdot \mathrm{s} / \mathrm{cm}^{-5} / \mathrm{m}^{2}\right) \text {. }
$$

The hemodynamic parameters corresponding to the 5-minute-long ECG waveforms obtained before TD were averaged. However, only the hemodynamic parameters with the nadir of the MAP were taken as the data for $10 \mathrm{~min}$ after TD. The percent change in the MAP after TD was defined as follows:

$$
\frac{\text { minimum MAP during } 10 \text { minutes after } T D-\text { averaged } M A P \text { for } T 4}{\text { averaged } M A P \text { for } T 4}(\%) \text {. }
$$

\section{Sample size calculation}

The primary outcome of this study was the sympathovagal balance before TD. In a pilot study using 10 patients, the results of the simple linear regression analysis to predict the percent change in MAP after TD according to the sympathovagal balance before TD showed the coefficient of determination $\left(\mathrm{R}^{2}\right)$ of 0.18 , the regression coefficient of 0.05 , and the standard deviation of sympathovagal balance before TD of 48.8. Based on these results, 52 patients were required to achieve $90 \%$ statistical power at a significance level of 0.05 for detecting a change in slope from 0.00 under the null hypothesis to 0.05 under the alternative hypothesis. Considering a dropout rate of $10 \%$, the sample size was set at 58 patients. The sample size was calculated using PASS 15 Power Analysis and Sample Size Software (2017) (NCSS, LLC. Kaysville, UT, USA, ncss.com/software/pass).

\section{Statistical analysis}

The data are presented as the mean \pm standard deviation or mean (minimum to maximum) for normally distributed data, median $\left(1^{\text {st }}-3^{\text {rd }}\right.$ quartile) for non-normally distributed data, and number of patients (percentage). The assumption of normality was tested by the Shapiro-Wilk's test. Simple linear and cubic regression analyses were performed to evaluate the effects of the sympathovagal balance before TD on the percent change in MAP after TD in all patients. Simple linear regression analysis was performed respectively in patients with and without hypotension after TD (hypotensive and non-hypotensive patients). The simple linear regression coefficients were compared using a $t$-test between the hypotensive and nonhypotensive patients (Weaver and Wuensch 2013). The effects of the sympathovagal balance before TD on the percent change in MAP after TD were also assessed controlling for the independent variables [age (Jacobsen et al. 1986), spinal anesthesia-induced hypotension, and duration of TI (Girardis et al. 2000, Jacobsen et al. 1986)], which might affect the percent change in MAP, by multiple linear regression analysis. Variance inflation factor $>5$ or a condition number $>10$ indicated the presence of multicollinearity (Kim 2019). The final regression model was built with the exclusion of multicollinear independent variables and a difference of less than 0.1 between the coefficient of determination $\left(R^{2}\right)$ and the adjusted $R^{2}$.

To analyze the longitudinal change in HRV and hemodynamic parameters, repeated measures analysis of variance was used. For post hoc multiple comparisons, paired $t$-tests were performed. The probability values were adjusted with Bonferroni correction. A two-tailed probability value $<0.05$ was considered statistically significant. The statistical analysis was performed using NCSS 2019 Statistical Software (2019) (NCSS, LLC., ncss.com/software/ncss) and IBM SPSS, version 19.0.0 (IBM Corp., Armonk, NY, USA).

\section{Results}

Four patients were excluded from the analysis: spinal anesthesia was converted to general anesthesia in one patient; unexpected arrhythmia developed in three patients whose preoperative ECG showed normal sinus rhythm. The mean age of the analyzed patients was 69 years. The tourniquet was inflated for $32 \mathrm{~min}$ on average (Table 1).

Following TD, the MAP of T5 was significantly reduced compared with those of $\mathrm{T} 1$ to $\mathrm{T} 4(\mathrm{P}<0.01)$ in all patients (Fig. 1A). The mean difference in MAP between $\mathrm{T} 4$ and $\mathrm{T} 5$ and its range were -11.6 (-46.9 to 3.4$) \mathrm{mm} \mathrm{Hg}$. The mean percent change in the MAP after TD and its range were -12.1 (-47.9 to 3.0) \%. Upon TI, the heart rate decreased from $72.5 \pm 12.8$ beats $/ \mathrm{min}$ (T1) and $70.1 \pm 14.7$ beats/min (T2) to $65.5 \pm 12.4$ beats/min (T3) and $64.8 \pm 12.0$ beats $/ \mathrm{min} \quad$ (T4) $\quad(\mathrm{P}<0.001, \quad$ Fig. $1 \mathrm{~A}) . \quad$ The reduction was followed by an increase to $68.8 \pm 12.6$ beats/min (T5) with $\mathrm{TD}(\mathrm{P}<0.001$ compared to 
Table 1. Demographic and perioperative data.

\begin{tabular}{lc}
\hline Variable & Value \\
\hline Age (years) & $69.4 \pm 5.7$ \\
Height $(\mathrm{cm})$ & $155.6 \pm 6.6$ \\
Weight $(\mathrm{kg})$ & $58.0 \pm 9.7$ \\
Side of surgery (right/left) & $28(51.9) / 26(48.2)$ \\
Level of lumbar puncture (L3 - 4/L4 - 5/L5 - S1) & $20(37.0) / 28(51.9) / 6(11.1)$ \\
Type of approach for spinal anesthesia (midline/paramedian) & $42(77.8) / 12(22.2)$ \\
Dose of 0.5\% hyperbaric bupivacaine for spinal anesthesia $(m g)$ & $11(11-12)$ \\
Duration of anesthesia (min) & $131.3 \pm 17.1$ \\
Total amount of fluid until tourniquet deflation (ml) & $625(387.5-850)$ \\
Duration of tourniquet inflation (min) & $32.5 \pm 5.6$ \\
Duration of surgery (min) & $90.8 \pm 10.1$ \\
\hline
\end{tabular}

Data are presented as mean $\pm \mathrm{SD}$, median $\left(1^{\text {st }}-3^{\text {rd }}\right.$ quartiles $)$, or number of patients (\%).
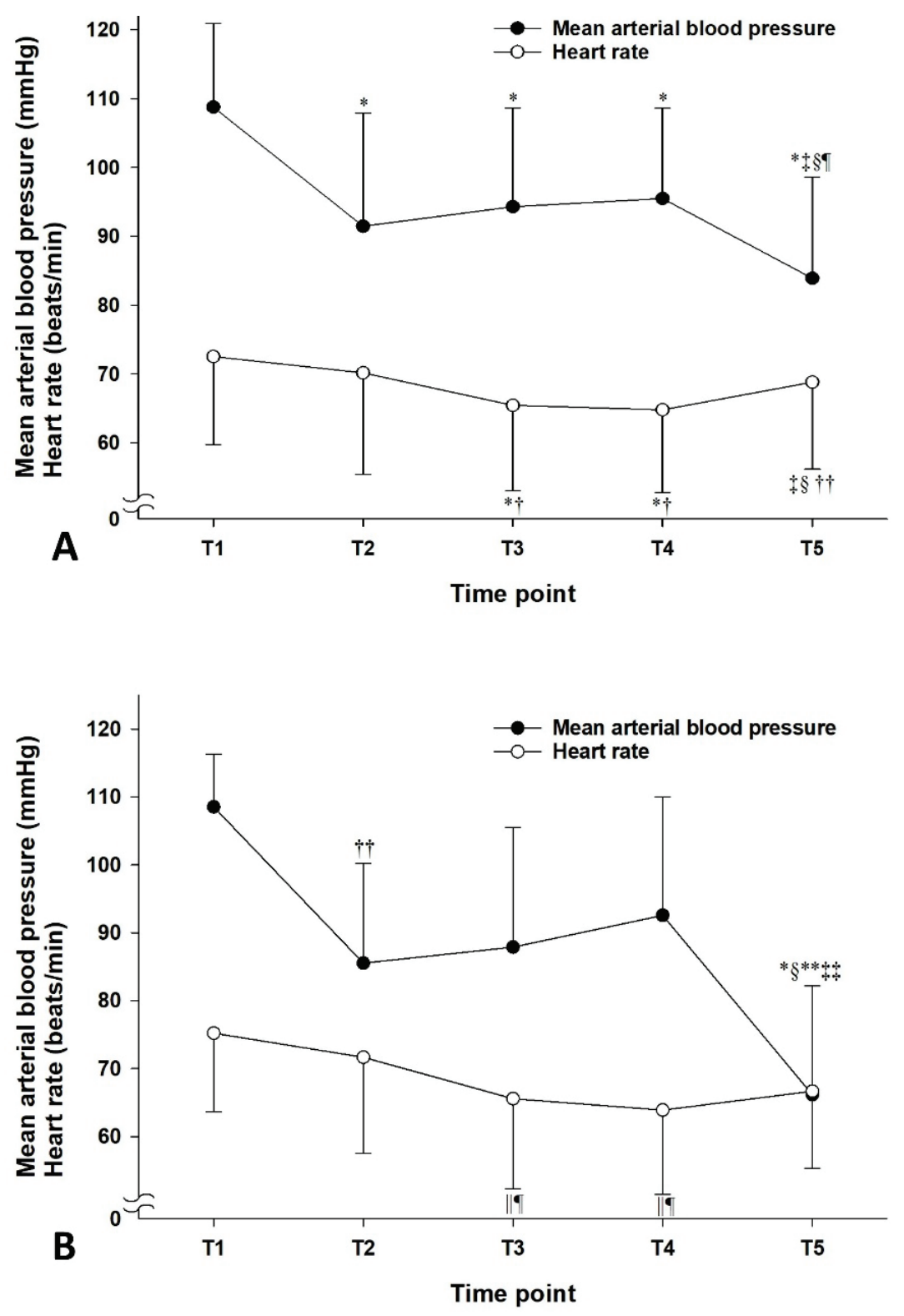

Fig. 1. Changes in arterial mean blood pressure and heart rate in all patients $(\mathbf{A} ; \mathrm{n}=54)$ and patients with hypotension after tourniquet deflation $(\mathbf{B} ; \mathrm{n}=9$ ). Data are presented as mean with SD. T1: during $5 \mathrm{~min}$ before the lateral position for spinal anesthesia, T2: between 15 and $20 \mathrm{~min}$ after the end of local anesthetic injection into the subarachnoid space, T3: between 10 and 15 min after tourniquet inflation, T4: during $5 \mathrm{~min}$ before tourniquet deflation, T5: nadir within $10 \mathrm{~min}$ after tourniquet deflation. ${ }^{*} \mathrm{P}<0.001$ compared to $\mathrm{T} 1 ;{ }^{+} \mathrm{P}<0.001$ compared to T2; ${ }^{\ddagger} \mathrm{P}<0.001$ compared to $\mathrm{T} 3{ }^{\S}{ }^{\S} \mathrm{P}<0.001$ compared to $\mathrm{T} 4 ;$ " $\mathrm{P}<0.01$ compared to $\mathrm{T} 1 ;$ " $\mathrm{P}<0.01$ compared to T2; ** $\mathrm{P}<0.01$ compared to $\mathrm{T} 3 ;{ }^{++} \mathrm{P}<0.05$ compared to $\mathrm{T} 1 ;{ }^{\neq \neq} \mathrm{P}<0.05$ compared to $\mathrm{T} 2$. 
T3 and T4). In hypotensive patients, the MAP also decreased after TD compared to $\mathrm{T} 1$ to $\mathrm{T} 4$ (Fig. 1B). However, the heart rate was not significantly changed after TD. A shift in sympathovagal balance toward sympathetic predominance (an increase in LF/HF) before TD was associated with a reduction in MAP after TD in the simple linear regression models for all patients and hypotensive patients $\left(\mathrm{R}^{2}=0.323, \mathrm{P}<0.001\right.$ and $\mathrm{R}^{2}=0.743$, $\mathrm{P}=0.003$, respectively, Fig. $2 \mathrm{~A}, 2 \mathrm{~B})$ and in the cubic regression model for all patients $\left(\mathrm{R}^{2}=0.371, \mathrm{P}<0.001\right.$, Fig. 2C). However, the association was not observed in the simple linear regression model for the nonhypotensive patients $\left(\mathrm{R}^{2}=0.037, \mathrm{P}=0.203\right.$, Fig. $\left.2 \mathrm{~B}\right)$. Accordingly, the simple linear regression coefficients were significantly different between the hypotensive and non-hypotensive patients [the difference in regression coefficient $(95 \%$ confidence interval $)=0.043(0.020$ to $0.066), \mathrm{P}<0.001]$. The association between sympathovagal balance before TD and change in MAP after TD was also observed in the multiple linear regression model. However, the effects of age, spinal anesthesia-induced hypotension, and duration of TI were insignificant (Table 2). TD significantly reduced TPRI from $3181.5 \pm 691.1$ dyne $\cdot \mathrm{s} / \mathrm{cm}^{-5} / \mathrm{m}^{2} \quad$ (T4) to $2602.3 \pm$ 633.2 dyne $\cdot \mathrm{s} / \mathrm{cm}^{-5} / \mathrm{m}^{2}$ (T5) in all patients (Fig. 3A) and from $3128.9 \pm 816.2$ dyne $\cdot \mathrm{s} / \mathrm{cm}^{-5} / \mathrm{m}^{2}$ (T4) to $2062.7 \pm$ 484.2 dyne $\cdot \mathrm{s} / \mathrm{cm}^{-5} / \mathrm{m}^{2}$ (T5) in hypotensive patients (Fig. 3B). Accordingly, cardiac index increased from $2.5 \pm 0.5 \mathrm{ml} / \mathrm{m}^{2}$ (T3 and T4) to $2.6 \pm 0.5 \mathrm{ml} / \mathrm{m}^{2}$ (T5) in all patients (Fig. 3A). However, no significant changes in cardiac index were observed in hypotensive patients (Fig. 3B). According to the HRV spectrograms (Fig. 4), a non-hypotensive patient with an age of 75 years (a decrease in MAP by $1 \%$ and an increase in LF/HF by $29 \%$ ) has a high degree of HRV (spectrogram dominant in red over blue) compared to that of a hypotensive patient with an age of 67 years (a decrease in MAP by $29 \%$ and an increase in LF/HF by $100 \%$ ).
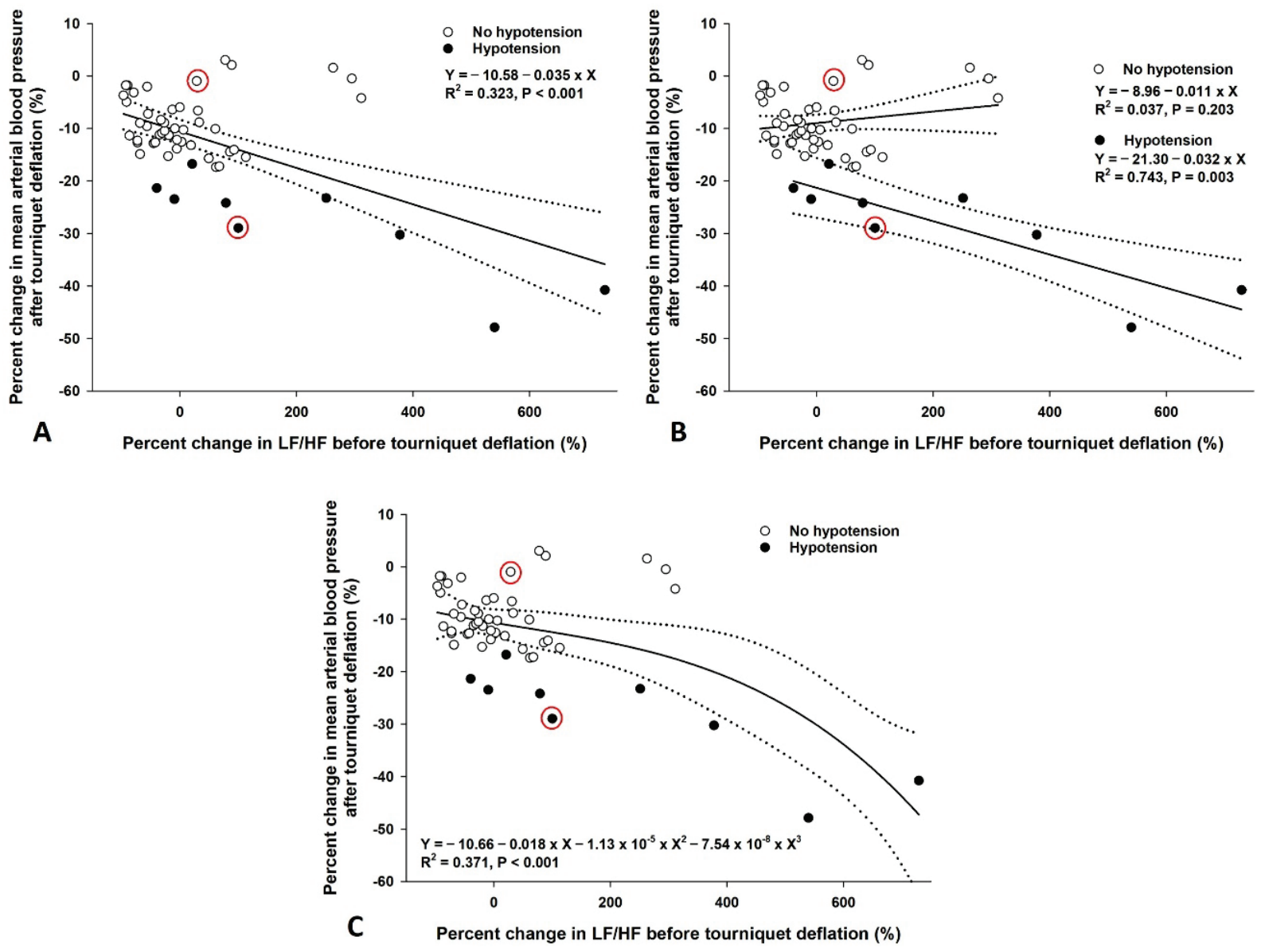

Fig. 2. Regression models between sympathovagal balance before tourniquet deflation and percent change in mean arterial blood pressure after tourniquet deflation. (A) Simple linear regression model for all patients $(n=54)$, (B) Simple linear regression models for patients with $(n=9)$ and without hypotension $(n=45)$ after tourniquet deflation, (C) Cubic regression model for all patients $(n=54)$. The solid line and 2 dashed lines represent the predictive values and their $95 \%$ confidence intervals. The two red circles indicate the data of two patients whose spectrograms were presented in Fig. 4. LF/HF: low-to-high frequency ratio of heart rate variability. 
Table 2. Multiple linear regression model to predict the percent change in mean arterial blood pressure following tourniquet deflation.

Predictive variable
Partial regression coefficient (95\% Confidence interval)
P-value

$\begin{array}{cc}-0.035(-0.049,-0.020) & <0.001 \\ 0.184(-0.221,0.588) & 0.365 \\ -0.791(-5.828,4.245) & 0.754 \\ 0.069(-0.343,0.482) & 0.738 \\ -25.342(-57.424,6.741) & 0.119\end{array}$

Coefficient of determination $\left(R^{2}\right)=0.340$, adjusted $R^{2}=0.286, P<0.001$. LF/HF, low-to-high frequency ratio of heart rate variability. No multicollinearity was detected.

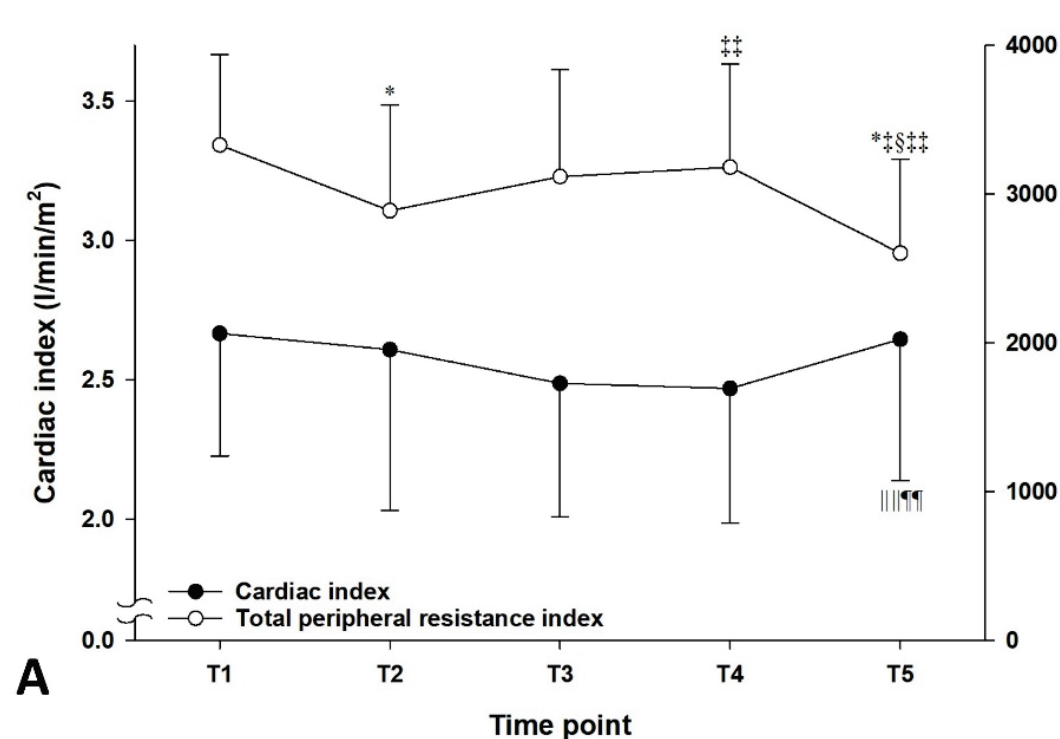

Percent change in $\mathrm{LF} / \mathrm{HF}$

Age (years)

Spinal anesthesia-induced hypotension

Duration of tourniquet inflation (min)

Intercept
Fig. 3. Changes in cardiac index and total peripheral resistance index in all patients $(\mathbf{A} ; n=54)$ and patients with hypotension after tourniquet deflation $(\mathbf{B} ; \mathrm{n}=9)$. Data are presented as mean with SD. T1: during $5 \mathrm{~min}$ before the lateral position for spinal anesthesia, T2: between 15 and 20 min after the end of local anesthetic injection into the subarachnoid space, T3: between 10 and $15 \mathrm{~min}$ after tourniquet inflation, T4: during $5 \mathrm{~min}$ before tourniquet deflation, T5: nadir within $10 \mathrm{~min}$ after tourniquet deflation. ${ }^{*} \mathrm{P}<0.001$ compared to $\mathrm{T} 1 ;{ }^{*} \mathrm{P}<0.001$ compared to T3; ${ }^{\S} \mathrm{P}<0.001$ compared to $\mathrm{T} 4 ; \quad$ II $\mathrm{P}<0.01$ compared to $\mathrm{T} 1$; ** $\mathrm{P}<0.01$ compared to T3; ${ }^{\ddagger \neq} \mathrm{P}<0.05$ compared to $\mathrm{T} 2 ; \quad$ II $\| \mathrm{P}<0.01$ compared to $\mathrm{T} 4$; ๆ ๆ $\mathrm{P}<0.05$ compared to T3.

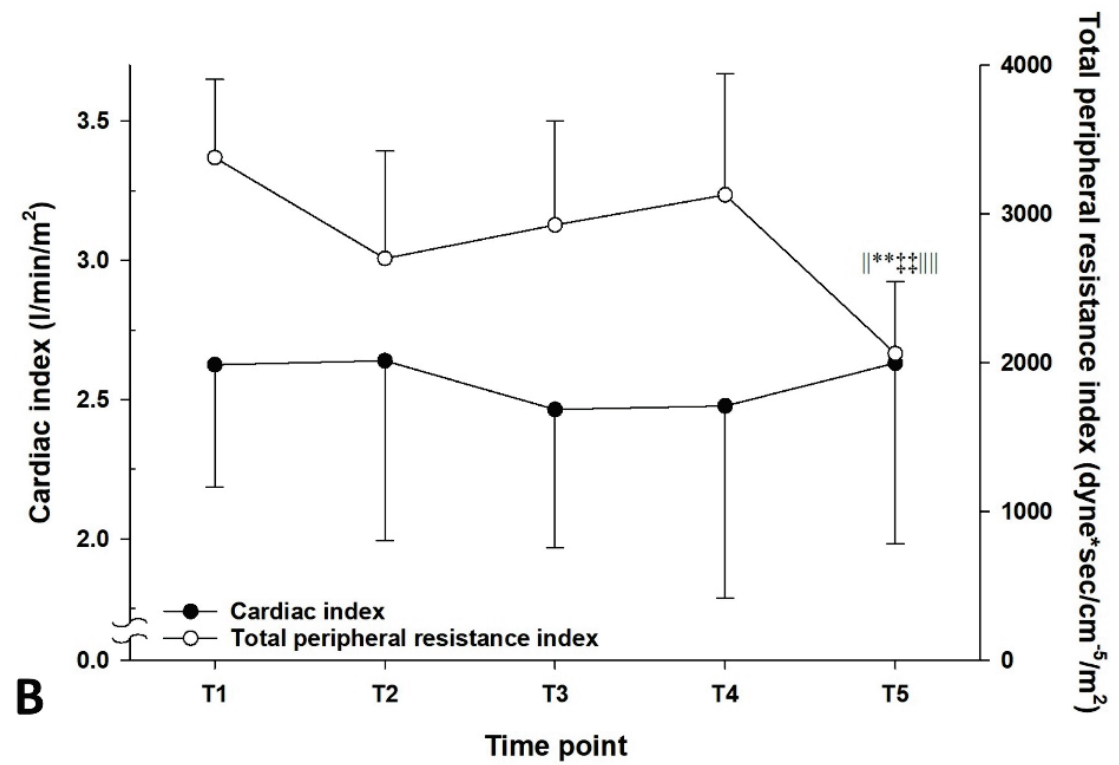



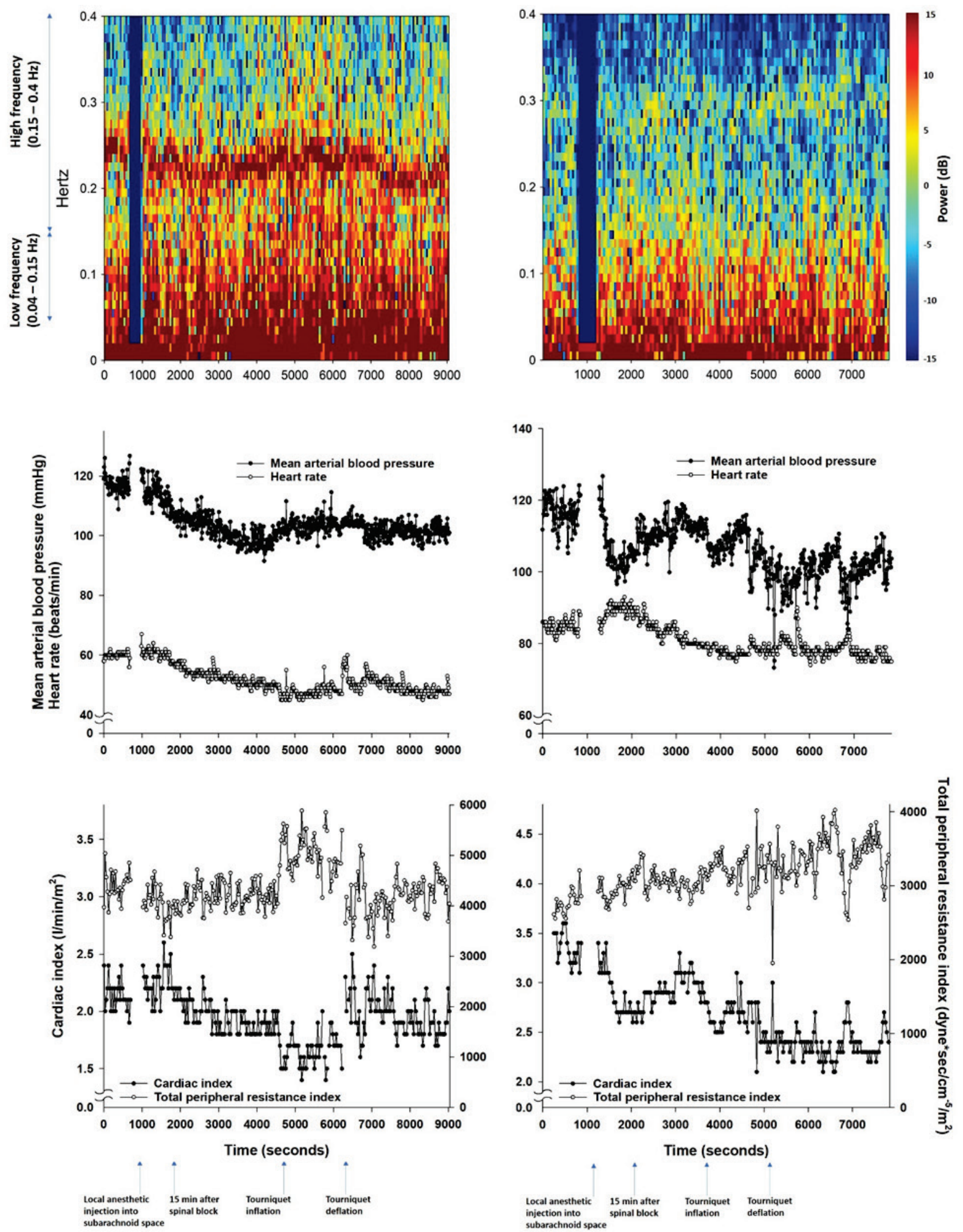

Fig. 4. Longitudinal changes in heart rate variability parameters, mean arterial blood pressure, heart rate, cardiac index, and total peripheral resistance index during the entire surgery in a patient who developed hypotension after tourniquet deflation (right panel) and one who did not (left panel).

Seventeen $(31.5 \%)$ and nine $(16.7 \%)$ patients experienced hypotension after the induction of spinal anesthesia and TD, respectively. Five patients $(9.3 \%)$ had two episodes of hypotension induced by both spinal anesthesia and TD. All episodes of hypotension were treated with only $10 \mathrm{mg}$ of ephedrine, except for one case, which was managed with $30 \mathrm{mg}$ of ephedrine for spinal anesthesia-induced hypotension. 


\section{Discussion}

In the present study, a shift in sympathovagal balance toward sympathetic predominance before TD was found to be associated with a reduction in ABP after TD. The reduction in ABP was accompanied by a decrease in TPRI and an increase in cardiac index. The mechanisms for the reduction in ABP are redistribution of the intravascular volume, which shifts from a lower limb to its proximal part by exsanguination before TI, to a reperfused lower limb; subsequent hemorrhage; and post-ischemic reactive hyperemia causing vasodilatation (Girardis et al. 2000, Gupta et al. 2008). However, their effects cannot be offset by an increase in cardiac index (Girardis et al. 2000). Hence, we hypothesize that the assessment of autonomic nervous activity which determines vascular resistance (total peripheral resistance [TPR]) is helpful for predicting a change in ABP after TD (Joyner et al. 2010).

When ABP was lowered in the elderly using trimethaphan which reduces TPR by selectively blocking ganglionic nicotinic receptors expressed in both parasympathetic and sympathetic ganglia (Julius et al. 1982), the baseline muscle sympathetic nerve activity and plasma norepinephrine level were correlated with the extent of reductions in TPR (Barnes et al. 2014) and ABP (Barnes et al. 2014, Jones et al. 2001). Similarly, under spinal anesthesia, which causes sympathectomy leading to a reduction in TPR (Butterworth 1998), the extent of a shift in baseline sympathovagal balance toward sympathetic predominance was correlated with that of a reduction in ABP (Hanss et al. 2006). In accordance with these findings, our study showed a correlation between the extent of a shift in sympathovagal balance toward sympathetic predominance before TD and that of a reduction in ABP measured after TD, which also decreases TPR. Although the mechanisms are different from each other, the hypotensive response (with reduced TPR) to the above three hemodynamic conditions was preceded by a sympathetic predominant state. In this regard, we speculate that if ABP is highly dependent on autonomic support (enhanced sympathetic tone), blunting or abolishment of the autonomic support would significantly reduce the ABP (Barnes et al. 2014, Jones et al. 2001).

We built a linear regression model to find the relationship between sympathovagal balance before TD and MAP after TD. Although statistically significant predictive performance was achieved by the models built from the data of all the patients and hypotensive patients, the model for non-hypotensive patients did not show the significant predictive performance implying that the hemodynamic response to the loss of autonomic support is different between the hypotensive and non-hypotensive patients. In addition, non-linear relationship was observed in previous studies between autonomic nervous activity and MAP (Behuliak et al. 2018, Greenway et al. 1967). Therefore, we created a non-linear (cubic) regression model that optimally fits the data from both groups of patients. According to the cubic regression model, less changes in sympathovagal balance, which are observed in most non-hypotensive patients, contribute to less changes in MAP. However, the more a shift in sympathovagal balance goes toward sympathetic predominance, the more the MAP decreases. Hence, the cubic regression model supports the above speculation that patients whose ABP is highly dependent on autonomic support develop hypotension after TD that withdraws the autonomic support.

Because autonomic nervous activity before TD does not solely affect the ABP after TD, multiple linear regression analysis was performed to assess the effect of autonomic nervous activity, taking into account the candidate risk factors [age (Jacobsen et al. 1986), long duration of TI (Girardis et al. 2000, Jacobsen et al. 1986), and spinal anesthesia-induced hypotension], which could not be controlled but influence systemic hemodynamics after TD. The final regression model showed the significant effect of autonomic nervous activity on a reduction in ABP after TD. However, no significant effects of the candidate risk factors were found.

It would be clinically useful to early detect patients developing hypotension by using HRV parameters. However, we assessed autonomic nervous activity immediately before TD limiting the time to prepare for anticipated hypotension after identifying patients at risk of hypotension. One previous study that investigated HRV parameters in patients undergoing general anesthesia could not find significant relationships between the preoperative HRV parameters and the extent of a reduction in MAP after TD (Huh et al. 2012). We assume that the absence of the relationships is attributed to unpredictability of changes in autonomic nervous activity after anesthesia induction. In the present study, sympathectomy resulting from spinal anesthesia can lead to hypotension, which is followed by administration of sympathomimetic agents, such as ephedrine. Because spinal anesthesia (Hanss et al. 2006) and ephedrine (Chen 
et al. 2010) affect autonomic nervous activity, the autonomic nervous activity altered by them renders preoperatively assessed HRV parameters unreliable. In addition, there might be unknown or known confounding factors [age (Jandackova et al. 2016), underlying hypertension (Singh et al. 1998), duration of TI (Girardis et al. 2000, Jacobsen et al. 1986) etc.] that keep affecting autonomic nervous activity until TD. Therefore, we used the HRV parameters immediately before TD as a primary outcome, which were assumed to reflect integrative effects of both known and unknown confounding factors on autonomic nervous activity until TD.

The low incidence of hypotension after TD [nine cases $(16.7 \%)$ ] precludes a comparison of LF/HF and the other variables between groups with and without hypotension. It is speculated that the relatively short duration of tourniquet application ( $32 \mathrm{~min}$ on average) compared with those of other studies (Huh et al. 2012, Jacobsen et al. 1986), which were approximate to or more than an hour, contributed to the low incidence of hypotension. Therefore, alternatively, we compared the HRV spectrograms from one patient who experienced hypotension after TD and another who did not. The hypotensive patient with an increased LF/HF before TD had HRV lower than the non-hypotensive patient (Fig. 4), implying that the active response of the autonomic nervous system to hemodynamic challenges offsets their negative effects.

Some limitations should be considered in this study. Because of high prevalence of hypertension (Calloway et al. 2014) and high female ratio (Cho et al. 2011) in patients undergoing total knee arthroplasty, we could not recruit patients with no underlying diseases nor balance the sex ratio in the subjects. Therefore, the effects of hypertension and sex on changes in the MAP after TD could not be excluded (Plevkova et al. 2020, Rauchova et al. 2020). Although LF/HF has gained wide acceptance (Malliani et al. 1991), its interpretation has limitations. LF can be influenced by parasympathetic activity (Houle and Billman 1999), and vice versa (Taylor et al. 2001). Furthermore, although both components of the autonomic nervous system do not change reciprocally (Billman 2013), a simple ratio value (LF/HF) was used. Nonetheless, LF/HF successfully predicted hemodynamic instability in various clinical situations (Barnes et al. 2014, Hanss et al. 2006, Jones et al. 2001). Regrettably, the observational setting of this study allows to find only associations but not cause-and-effect relationships between variables, which are usually investigated in a randomized controlled trial (Sibbald and Roland 1998). Hence, only the speculations, not the clear explanations, were provided about the relationships between sympathovagal balance before TD and a reduction in ABP after TD. Although statistically significant changes in heart rate were found throughout the study period, their clinical impacts seem insignificant because changes in heart rate by less than 10 beats/min within a normal range generally do not reflect significant changes in patients' clinical conditions. Lastly, we did not assess postoperative outcomes because the assessment was complicated by contralateral surgery performed two weeks after the first surgery based on our institution's protocol.

\section{Conclusions}

In conclusion, a shift in sympathovagal balance toward sympathetic predominance immediately before TD is associated with a reduction in ABP after TD. Therefore, our results are expected to help clinicians prepare for hemodynamic instability following TD. However, further study is warranted to find useful clinical maneuvers to make more favorable autonomic nervous activity before TD for the prevention of hypotension following TD.

\section{Conflict of Interest}

There is no conflict of interest.

\section{Acknowledgements}

This work was supported by the Research Institute of Medical Science, Daegu Catholic University (201602).

\section{References}

BARNES JN, HART EC, CURRY TB, NICHOLSON WT, EISENACH JH, WALLIN BG, CHARKOUDIAN N, JOYNER MJ: Aging enhances autonomic support of blood pressure in women. Hypertension 63: 303-308, 2014. https://doi.org/10.1161/HYPERTENSIONAHA.113.02393

BEHULIAK M, BENCZE M, POLGAROVA K, KUNES J, VANECKOVA I, ZICHA J: Hemodynamic response to gabapentin in conscious spontaneously hypertensive rats. Hypertension 72: 676-685, 2018. https://doi.org/10.1161/HYPERTENSIONAHA.118.09909 
BILLMAN GE: The LF/HF ratio does not accurately measure cardiac sympatho-vagal balance. Front Physiol 4: 26, 2013. https://doi.org/10.3389/fphys.2013.00026

BUTTERWORTH J: Physiology of spinal anesthesia: what are the implications for management? Reg Anesth Pain Med 23: 370-373, 1998.

CALLOWAY JJ, MEMTSOUDIS SG, KRAUSER DG, MA Y, RUSSELL LA, GOODMAN SM: Hemodynamic effects of angiotensin inhibitors in elderly hypertensives undergoing total knee arthroplasty under regional anesthesia. J Am Soc Hypertens 8: 644-651, 2014. https://doi.org/10.1016/j.jash.2014.05.017

CHARKOUDIAN N, JOYNER MJ, JOHNSON CP, EISENACH JH, DIETZ NM, WALLIN BG: Balance between cardiac output and sympathetic nerve activity in resting humans: role in arterial pressure regulation. J Physiol 568: 315-321, 2005. https://doi.org/10.1113/jphysiol.2005.090076

CHARKOUDIAN N, RABBITTS JA: Sympathetic neural mechanisms in human cardiovascular health and disease. Mayo Clin Proc 84: 822-830, 2009. https://doi.org/10.1016/S0025-6196(11)60492-8

CHEN WL, TSAI TH, YANG CC, KUO TB: Acute effects of ephedra on autonomic nervous modulation in healthy young adults. Clin Pharmacol Ther 88: 39-44, 2010. https://doi.org/10.1038/clpt.2010.66

CHO HJ, CHANG CB, KIM KW, PARK JH, YOO JH, KOH IJ, KIM TK: Gender and prevalence of knee osteoarthritis types in elderly Koreans. J Arthroplasty 26: 994-999, 2011. https://doi.org/10.1016/j.arth.2011.01.007

COURTOIS M, FATTAL PG, KOVACS SJ JR, TIEFENBRUNN AJ, LUDBROOK PA: Anatomically and physiologically based reference level for measurement of intracardiac pressures. Circulation 92: 1994-2000, 1995. https://doi.org/10.1161/01.cir.92.7.1994

FURLAN R, HEUSSER K, MINONZIO M, SHIFFER D, CAIRO B, TANK J, JORDAN J, DIEDRICH A, GAUGER P, ZAMUNER AR, DIPAOLA F, PORTA A, BARBIC F: Cardiac and vascular sympathetic baroreflex control during orthostatic pre-syncope. J Clin Med 8: 1434, 2019. https://doi.org/10.3390/jcm8091434

GIRARDIS M, MILESI S, DONATO S, RAFFAELLI M, SPASIANO A, ANTONUTTO G, PASQUALUCCI A, PASETTO A: The hemodynamic and metabolic effects of tourniquet application during knee surgery. Anesth Analg 91: 727-731, 2000. https://doi.org/10.1097/00000539-200009000-00043

GREENWAY CV, LAWSON AE, MELLANDER S: The effects of stimulation of the hepatic nerves, infusions of noradrenaline and occlusion of the carotid arteries on liver blood flow in the anaesthetized cat. J Physiol 192: 21-41, 1967. https://doi.org/10.1113/jphysiol.1967.sp008285

GUPTA K, AGGARWAL N, RAO M, VERMA UC, ANAND R: Re-emphasizing the importance of tourniquet time: severe myocardial depression following tourniquet deflation. Acta Anaesthesiol Scand 52: 873, 2008. https://doi.org/10.1111/j.1399-6576.2008.01663.x

HANSS R, BEIN B, WESELOH H, BAUER M, CAVUS E, STEINFATH M, SCHOLZ J, TONNER PH: Heart rate variability predicts severe hypotension after spinal anesthesia. Anesthesiology 104: 537-545, 2006. https://doi.org/10.1097/00000542-200603000-00022

HARRIS FJ: On the use of windows for harmonic analysis with the discrete Fourier transform. Proc IEEE 66: 51-83, 1978. https://doi.org/10.1109/PROC.1978.10837

Heart Rate Variability: standards of measurement, physiological interpretation and clinical use. Task Force of the European Society of Cardiology and the North American Society of Pacing and Electrophysiology. Circulation 93: 1043-1065, 1996.

HOULE MS, BILLMAN GE: Low-frequency component of the heart rate variability spectrum: a poor marker of sympathetic activity. Am J Physiol 276: H215-H223, 1999. https://doi.org/10.1152/ajpheart.1999.276.1.H215

HUH IY, KIM DY, LEE JH, SHIN SJ, CHO YW, PARK SE: Relation between preoperative autonomic function and blood pressure change after tourniquet deflation during total knee replacement arthroplasty. Korean J Anesthesiol 62: 154-160, 2012. https://doi.org/10.4097/kjae.2012.62.2.154

INCOGNITO AV, SAMORA M, CARTAFINA RA, GUIMARAES GMN, DAHER M, MILLAR PJ, VIANNA LC: Pharmacological assessment of the arterial baroreflex in a young healthy obese male with extremely low baseline muscle sympathetic nerve activity. Clin Auton Res 28: 593-595, 2018. https://doi.org/10.1007/s10286-018-0559-2

JACOBSEN J, RORSGAARD S, SECHER NH: Bradycardia during hypotension following release of a tourniquet in orthopaedic surgery. Acta Anaesthesiol Scand 30: 511-514, 1986. https://doi.org/10.1111/j.13996576.1986.tb02466.x 
JANDACKOVA VK, SCHOLES S, BRITTON A, STEPTOE A: Are changes in heart rate variability in middle-aged and older people normative or caused by pathological conditions? Findings from a large population-based longitudinal cohort study. J Am Heart Assoc 5: e002365, 2016. https://doi.org/10.1161/JAHA.115.002365

JONES PP, SHAPIRO LF, KEISLING GA, JORDAN J, SHANNON JR, QUAIFE RA, SEALS DR: Altered autonomic support of arterial blood pressure with age in healthy men. Circulation 104: 2424-2429, 2001. https://doi.org/10.1161/hc4501.099308

JOYNER MJ, CHARKOUDIAN N, WALLIN BG: A sympathetic view of the sympathetic nervous system and human blood pressure regulation. Exp Physiol 93: 715-724, 2008. https://doi.org/10.1113/expphysiol.2007.039545

JOYNER MJ, CHARKOUDIAN N, WALLIN BG: Sympathetic nervous system and blood pressure in humans: individualized patterns of regulation and their implications. Hypertension 56: 10-16, 2010. https://doi.org/10.1161/HYPERTENSIONAHA.109.140186

JULIUS S, SANCHEZ R, MALAYAN S, HAMLIN M, ELKINS M, BRANT D, BOHR DF: Sustained blood pressure elevation to lower body compression in pigs and dogs. Hypertension 4: 782-788, 1982. https://doi.org/10.1161/01.hyp.4.6.782

KAIMAR P, SANJI N, UPADYA M, MOHAMMED KR: A comparison of hypotension and bradycardia following spinal anesthesia in patients on calcium channel blockers and beta-blockers. Indian J Pharmacol 44: 193-196, 2012. https://doi.org/10.4103/0253-7613.93847

KIM JH: Multicollinearity and misleading statistical results. Korean J Anesthesiol 72: 558-569, 2019. https://doi.org/10.4097/kja.19087

MALLIANI A, PAGANI M, LOMBARDI F, CERUTTI S: Cardiovascular neural regulation explored in the frequency domain. Circulation 84: 482-492, 1991. https://doi.org/10.1161/01.cir.84.2.482

MISEK J, VETERNIK M, TONHAJZEROVA I, JAKUSOVA V, JANOUSEK L, JAKUS J: Radiofrequency electromagnetic field affects heart rate variability in rabbits. Physiol Res 69: 633-643, 2020. https://doi.org/10.33549/physiolres.934425

ODINSSON A, FINSEN V: Tourniquet use and its complications in Norway. J Bone Joint Surg Br 88: 1090-1092, 2006. https://doi.org/10.1302/0301-620X.88B8.17668

PLEVKOVA J, BROZMANOVA M, HARSANYIOVA J, STERUSKY M, HONETSCHLAGER J, BUDAY T: Various aspects of sex and gender bias in biomedical research. Physiol Res 69 (Suppl 3): S367-S378, 2020. https://doi.org/10.33549/physiolres.934593

RAUCHOVA H, HOJNA S, KADLECOVA M, VANECKOVA I, ZICHA J: Sex differences in blood pressure of aged Ren-2 transgenic rats. Physiol Res 69: 245-252, 2020. https://doi.org/10.33549/physiolres.934369

SHENG Y, ZHU L: The crosstalk between autonomic nervous system and blood vessels. Int J Physiol Pathophysiol Pharmacol 10: 17-28, 2018.

SIBBALD B, ROLAND M: Understanding controlled trials. Why are randomised controlled trials important? BMJ 316 : 201, 1998. https://doi.org/10.1136/bmj.316.7126.201

SINGH JP, LARSON MG, TSUJI H, EVANS JC, O'DONNELL CJ, LEVY D: Reduced heart rate variability and newonset hypertension: insights into pathogenesis of hypertension: the Framingham Heart Study. Hypertension 32: 293-297, 1998. https://doi.org/10.1161/01.hyp.32.2.293

TAYLOR JA, MYERS CW, HALLIWILL JR, SEIDEL H, ECKBERG DL: Sympathetic restraint of respiratory sinus arrhythmia: implications for vagal-cardiac tone assessment in humans. Am J Physiol Heart Circ Physiol 280: H2804-H2814, 2001. https://doi.org/10.1152/ajpheart.2001.280.6.H2804

WALLIN BG, CHARKOUDIAN N: Sympathetic neural control of integrated cardiovascular function: insights from measurement of human sympathetic nerve activity. Muscle Nerve 36: 595-614, 2007. https://doi.org/10.1002/mus.20831

WEAVER B, WUENSCH KL: SPSS and SAS programs for comparing Pearson correlations and OLS regression coefficients. Behav Res Methods 45: 880-895, 2013. https://doi.org/10.3758/s13428-012-0289-7

WELCH P: The use of fast Fourier transform for the estimation of power spectra: A method based on time averaging over short, modified periodograms. IEEE Trans Audio Electroac 15: 70-73, 1967. https://doi.org/10.1109/TAU.1967.1161901 